\title{
Mechanical Behavior of Woven Composite Reinforcements While Forming
}

\author{
Gilles Hivet, J EAN LAUNAy, A LAIN GASSER, \\ JEAN LUC DANIEL AND PHILIPPE BOISSE \\ Laboratoire de Mécanique des Systèmes et des Procédés, ENSAM $151 \mathrm{Bd}$ \\ de l'Hôpital, 75013 Paris, France \& ESEM rue Leonard de Vinci, 45072 \\ Orléans, France
}

\begin{abstract}
Simulation of the forming processes of thin composite structures is necessary at the design level in order to check the feasibility of the shape and to know the position of the reinforcements. A finite element analysis of fabric shaping process needs the knowledge of the mechanical behavior of the woven reinforcement. This behavior is non linear because of the shape variation of the weaving pattern when it is loaded. Experimental results are obtained from biaxial tests in the case of balanced and unbalanced fabric. A constitutive model consistent with the geometry of the woven pattern is proposed. It is based on experimental results achieved by biaxial tensile tests. 3D simulations of the unit woven cell submitted to biaxial tensions are also performed and compared to experiments.
\end{abstract}

\section{INTRODUCTION}

W Hen manufacturing THIN composites, complex shapes can be formed using a single operation. Both thermoset and thermoplastic matrices can be used. Thermoset prepreg fabric can be draped before polymerization of the matrix. The resin transfer molding (RTM) process is an alternative approach enabling automation. A resin is injected into a fabric preform previously shaped by a deep drawing operation [1]. Some advantages of thermoplastic matrices include possible recycling and shorter process cycle times [2]. Plane plates made of thermoplastic resin and long fiber reinforcements can be shaped at high temperature [3]. These different

Author to whom correspondence should be addressed. E-mail: Philippe.Boisse@paris.ensam.fr 
forming processes are based on the large formability of fabrics. Constitutive models of fiber fabric are necessary when a finite element approach is used to simulate the forming process in order to check its feasibility. This is not the case when fishnet algorithms are used [4]. In these methods the results do not depend on the fabric behavior and it is a drawback of such approaches. The objective of this paper is to present a study of the mechanical behavior of the fiber fabrics used as composite reinforcements. Mechanics of fiber fabrics is a three-scale problem. At macroscopic scale, the fabric is considered as a continuous medium neighboring a surface in the 3D space. Finite element analyses and especially simulations of forming processes (which are our objective) are made at this scale. At an intermediate scale (mesoscopic), the change of shape of the elementary woven pattern leads to geometrical nonlinearities. This phenomenon is biaxial accounting for the weaving of warp and weft yarns. Finally the yarns are made of single filaments (microscopic scale). The shape of the yarn cross sections is mainly influenced by the laying out of the filaments and by their contact and friction during tension of the fabric. The fabric macroscopic behavior results from these phenomena at a lower scale that lead to a nonlinear behavior.

The first goal of the paper is to present an experimental study of the mechanical behavior under biaxial tension. Experimental results are obtained for a set of fiber fabric reinforcements that show the consequences of undulations and interactions at the mesoscopic scale. From those experimental results a constitutive model is written and identified. This model ensures the non-penetration of the yarns during the deformation contrary to other models $[5,6]$. This constitutive model can be used in the formulation of finite elements specific to fiber fabrics [7]. Two forming simulation examples using such elements are finally presented.

\section{FIBER FABRIC MECHANICAL BEHAVIOR}

The present study focuses on the forming stage of the RTM process (manufacture of the preform), consequently, the fiber fabrics under consideration are among those used in this process, mainly in the field of aeronautic applications. They do not include any resin (which is injected after the forming stage).

Studies about woven composites, i.e., matrix reinforced by fabrics, are numerous ([9] for example). Generally, they aim at defining the characteristics of an equivalent homogeneous material [10] and forecasting the matrix damage under high load [11]. In contrast, the mechanical behavior of dry fabrics is less studied [5,12]. The deformation modes of a fabric during a shaping process are very different of those of a metal blank during a sheet 
metal forming process. That is the reason why the numerical codes which are now very efficient for sheet metal forming, cannot be used in case of fabric forming. The specific behavior of woven reinforcements is a consequence of the possible motion between the yarns themselves and between the numerous small fibers constituting a yarn. The cross section of these fibers is very small and each fiber has a very weak bending and compressive stiffness. Consequently, these yarn properties are negligible in comparison to the tensile stiffness. Due to the possible rotations between warp and weft yarns, the dry fabric has no in-plane shear stiffness and large angular variations between warp and weft directions are allowed. Those variations are large $\left(45^{\circ}\right.$ in Figure 1) and can reach $60^{\circ}$ in some cases. This deformation is the principal mode that allows one to obtain doubly curved shapes. The evolution of a straight line grille drawn on the fabric prior to shaping is shown in Figure 1. The drawn lines become curved but remain continuous. Taking into account that these lines have been drawn alternatively on warp and weft yarns, it can be deduced that two initially superimposed yarns remain superimposed during the deformation process. One of the main consequences of this continuous behavior is the possibility to use a classic Lagrangian finite element approach for the surface domain of the fabric.

Global equilibrium on the initial configuration is classically written:

$$
\int_{\Omega_{0}} \mathbf{S}: \delta \mathbf{E}(\eta) d V_{0}-\int_{\Omega_{0}} \mathbf{f}_{0} \cdot \eta d V_{0}+\int_{\Gamma_{t_{0}}} \mathbf{t}_{0} \cdot \eta d A_{0}=0
$$

$\forall \eta$ virtual displacement equal to zero on $\Gamma_{u}$, part of the frontier with prescribed displacements. $\mathbf{f}_{0}$ are body loads in $\Omega_{0}$ and $\mathbf{t}_{0}$ are surface loads on $\Gamma_{t_{0}}$, part of the frontier with prescribed efforts. $\delta \mathbf{E}(\eta)$ is the variation of the Green-Lagrange deformation tensor $\mathbf{E}$ in the virtual displacement $\eta$. $\mathbf{S}$ denotes the second Piola-Kirchhoff stress tensor.

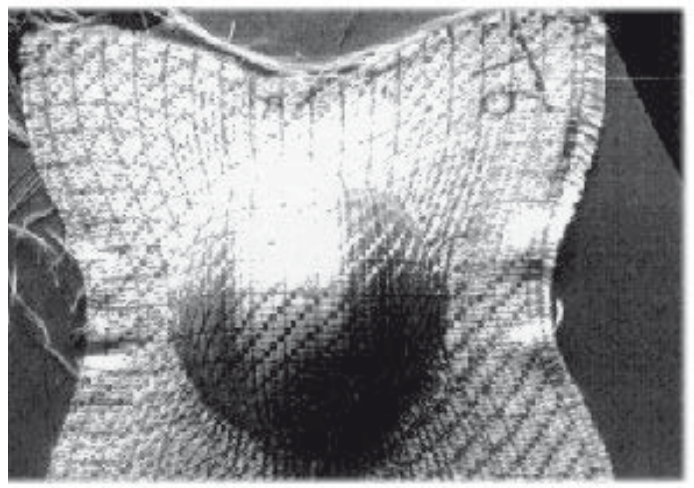

Figure 1. Deformation of the fabric [8]. 


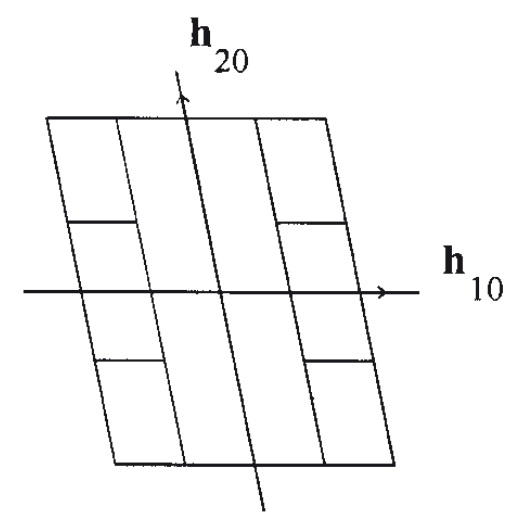

Figure 2. Elementary woven pattern.

Considering an elementary pattern in its initial position and denoting $\mathbf{h}_{10}$ and $\mathbf{h}_{20}$ the unit vectors in the warp and weft directions, respectively (Figure 2), and considering that the yarns have only tensile stiffness, the Lagrangian tensile tensor of second order $\mathbf{T}$ is defined by:

$$
\mathbf{T}=T^{11} \mathbf{h}_{10} \otimes \mathbf{h}_{10}+T^{22} \mathbf{h}_{20} \otimes \mathbf{h}_{20} \quad\left(T^{11} \text { and } T^{22} \geq 0\right)
$$

with

$$
T^{11}=\int_{A_{10}} S^{11} d A_{0}, \quad T^{22}=\int_{A_{20}} S^{22} d A_{0}
$$

where $A_{10}$ and $A_{20}$ are the cross sections of yarns directed by $\mathbf{h}_{10}$ and $\mathbf{h}_{20} . T^{11}$ and $T^{22}$ are the moduli of the efforts along warp and weft yarns. $n$ cell denotes the number of elementary cells of the structure. We note $\mathbf{W}$ the lagrangian tensor of second order like:

$$
{ }^{p} \mathbf{W}={ }^{p} W^{11}{ }^{p} \mathbf{h}_{10} \otimes{ }^{p} \mathbf{h}_{10}+{ }^{p} W^{22}{ }^{p} \mathbf{h}_{20} \otimes{ }^{p} \mathbf{h}_{20}
$$

with

$$
{ }^{p} W^{11}={ }^{p} T^{11 p} L_{0_{1}}, \quad{ }^{p} W^{22}={ }^{p} T^{22}{ }^{p} L_{0_{2}}
$$

The global equilibrium on the initial configuration can be written in the following simplified form [13]:

$$
\sum_{p=1}^{n \text { cell }}{ }^{p} \delta \mathrm{E}_{11}(\eta){ }^{p} W^{11}+{ }^{p} \delta \mathrm{E}_{22}(\eta)^{p} W^{22}-\int_{\Omega_{0}} \mathbf{f}_{0} \cdot \eta d V_{0}-\int_{\Gamma_{t_{0}}} \mathbf{t}_{0} \cdot \eta d A_{0}=0
$$

$\forall \eta / \eta=0$ on $\Gamma_{u}$ where $\mathbf{h}_{\alpha 0} \cdot \mathbf{h}^{\beta 0}=\delta_{\alpha}^{\beta}$ and ${ }^{p} \delta \mathbf{E}(\eta)={ }^{p} \delta \mathrm{E}_{\alpha \beta}(\eta){ }^{p} \mathbf{h}^{\alpha 0} \otimes{ }^{p} \mathbf{h}^{\beta 0}$ 


\section{BIAXIAL TENSILE TESTS}

In the previous approach the mechanical behavior of the fabric is defined by the biaxial relation between the weft and warp tension and strains. Consequently, a biaxial tensile device is used in order to analyze and identify the fabric behavior [6,14]. Presented in Figure 3, the device is made of two deformable parallelograms. When the system is compressed, it generates tensile deformation in each direction of the cross specimen put in the middle of the device. One of the parallelograms has adjustable dimensions, in order to set various deformation rates (the ratio is denoted by $k$ ). As stated previously, the major mechanism of deformation consists of the angular variation between the two weaving directions. So, a special system is adapted on the device to adjust the tensile angle between warp and weft yarns, in order to represent the real behavior of the fabric during the shaping process.

The cross shaped specimen is well adapted to the biaxial test of fabrics because of the lack of in-plane shear stiffness. Captors, set close to the specimen, are able to give tension and deformation in both directions. Strain measures are done by optical methods or by mechanical extensometers. The optical measurements check the homogeneity of the strain field within the active part of the specimen [15]. Both methods give equivalent global results. The load-strain curves deduced from biaxial tests are presented in Figure 4 for carbon fiber twill weave. This fabric is used in aeronautic applications. A single set of curves is shown because this fabric is balanced and the behaviors in warp and weft directions are equivalent. Due to undulation variations, a nonlinear behavior is observed at the beginning of the tensile test although the single carbon yarn has a linear response to a tensile loading. Moreover, this nonlinear characteristic is biaxial because

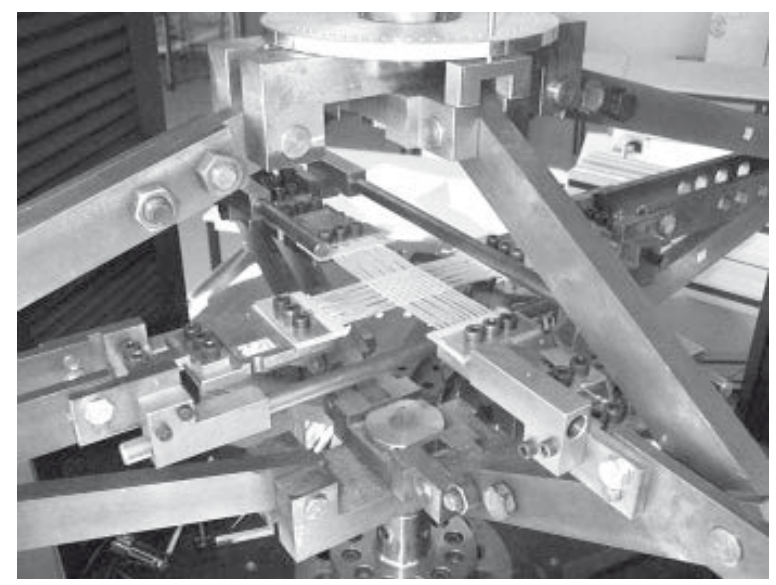

Figure 3. Biaxial tensile device and cross specimen. 


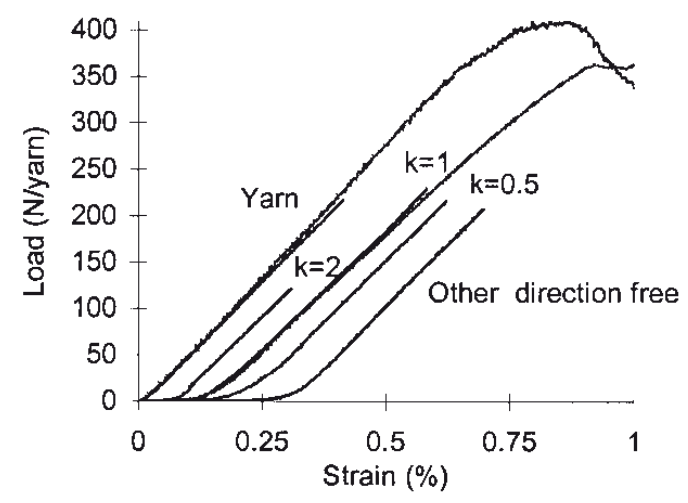

Figure 4. Results for a twill of carbon ( $k$ is the warp/weft strain ratio).
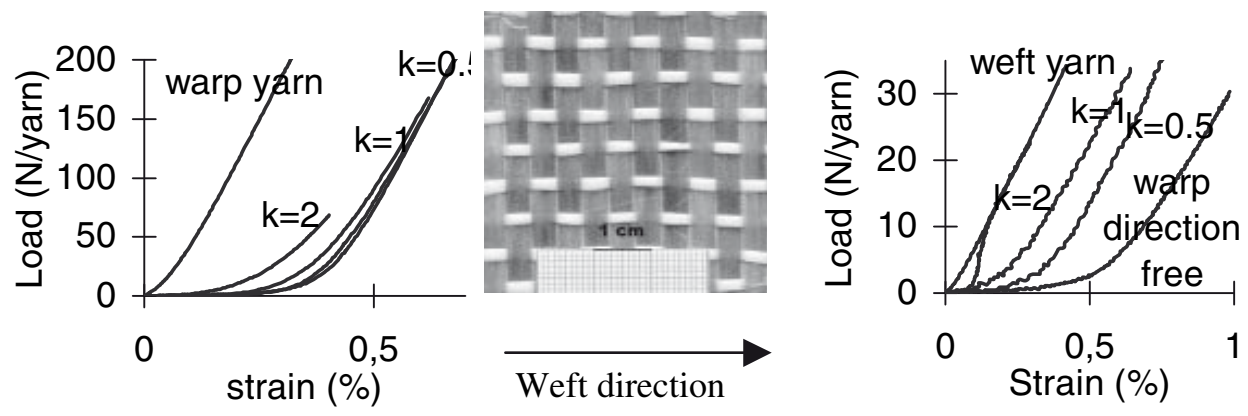

Figure 5. Biaxial tensile test on an unbalanced plain weave glass fiber fabric.

it depends on the deformation rate. Thus, the behavior of yarns in one direction is mainly influenced by the behavior of the yarns in the other direction. The test has been continued until the fracture for $k=1$. That permits one to notice that the nonlinear part of the behavior $(0.3 \%$ for the carbon twill) is important in comparison to the strain at fracture $(0.8 \%)$. A second experimental result is given Figure 5 in case of a glass plain weave fabric (also used in the field of aeronautic applications). This fabric is very unbalanced and the curves in warp and weft are very different. Because the stiffness of the yarn is much larger, the load/strain response in the warp direction is not much modified by the strain in weft direction. Nevertheless, the tensile behavior in the warp direction is much more non linear than those of the warp yarn. In the weft direction, the behavior of the fabric is mainly influenced by the warp strain ratio.

\section{CONSTITUTIVE MESO-MACRO MODEL CONSISTENT WITH THE GEOMETRY}

The aim of this model is to simulate the behavior of fabrics in a finite element code. It must therefore provide at the elementary yarn level the nonlinear behavior rules between weft and warp tensions according to weft 


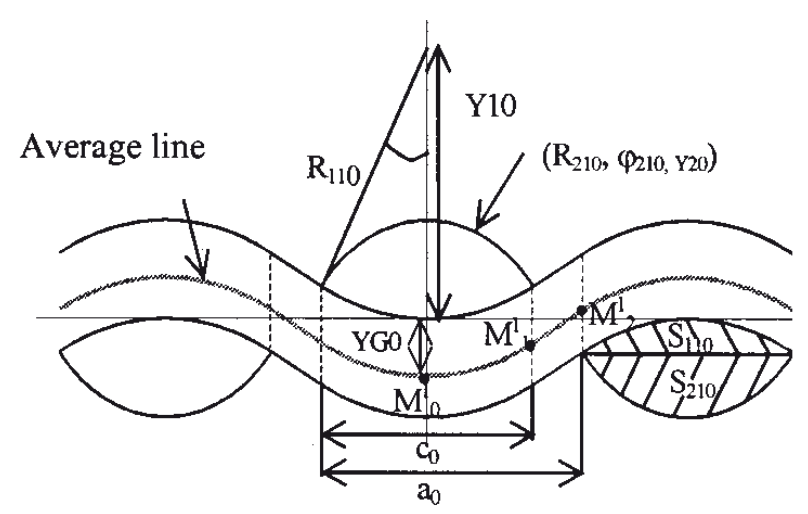

Figure 6. Transverse cut of the model.

and warp strains. The objective of the geometrically consistent model presented hereafter is to take into account the geometrical nonlinearities, which are mostly caused by undulation changes in the fabric at the local level, so that the meso-macro behavior rules can be identified. The thread is represented by its average line, which is described by a yarn type model. Each fiber is considered as a tube, the transverse section of which is described by two arcs with different radius. The geometrical parameters of this second model are given on Figure 6. The static equilibrium of the yarn gives tensions in the fibers directions as :

$$
T_{f}^{1}=-p R_{12} R_{11} \varphi_{12} \quad T_{f}^{2}=-p R_{11} R_{12} \varphi_{11}
$$

In the fabric directions tensions are then defined as: $T^{\alpha \alpha}=T_{f}^{\alpha} \cos \varphi_{1 \alpha}$ The thread distortions being light, the measure of the thread distortion is:

$$
\varepsilon_{f}^{\alpha}=\frac{\left(L_{\alpha}-L_{\alpha 0}\right)}{L_{\alpha 0}} \quad \varepsilon_{\alpha \alpha}=\frac{1}{2}\left(\frac{a_{\alpha}^{2}-a_{\alpha 0}^{2}}{a_{\alpha 0}^{2}}\right)
$$

Experiments [6,16], as well as the finite element models at the thread level [17] have shown the key role of fibers transverse strain in the undulation changes of the fabric. This confirms that the transverse behavior rule is crucial for the models under development. Experimental results also show that the compression rule depends on at least two parameters: the compression force undergone by the thread and the tension in the thread. This behavior rule is extremely difficult to verify experimentally, but various studies lead to an expression of the fabric transverse compression rule with three parameters [17]:

$$
\phi=\left|z_{1}-z_{2}\right|=A\left(2-e^{-B S p}-e^{-\tilde{B} /\left(T_{f}^{1}+T_{f}^{2}\right) / 2}\right), z_{\alpha} \text { displacement of } M_{0}^{\alpha}
$$


The traction response rules are given by the biaxial trials.

$$
T_{f}^{\alpha}=g_{\alpha}\left(\varepsilon_{f}^{\alpha}\right)
$$

The model geometry provides the last equations defining the model [6].

$$
\begin{aligned}
z_{\alpha}= & Y_{\alpha}+Y_{G 0}-\frac{1}{S_{1 \alpha}+S_{2 \alpha}}\left(S_{1 \alpha} R_{1 \alpha}\left(\frac{2 \sin ^{3}\left(\varphi_{1 \alpha}\right)}{3\left(\sin \left(\varphi_{1 \alpha}\right) \cos \left(\varphi_{1 \alpha}\right)-\varphi_{1 \alpha}\right)}+\cos \left(\varphi_{1 \alpha}\right)\right)\right) \\
& +S_{2 \alpha} R_{2 \alpha}\left(\frac{2 \sin ^{3}\left(\varphi_{2 \alpha}\right)}{3\left(-\sin \left(\varphi_{2 \alpha}\right) \cos \left(\varphi_{2 \alpha}\right)+\varphi_{2 \alpha}\right.}-\cos \left(\varphi_{2 \alpha}\right)\right)
\end{aligned}
$$

$$
L_{\alpha}=\frac{\varphi_{1 \alpha} a_{\alpha}\left(X_{\alpha 0}+1 / 2\right)}{\varphi_{1 \alpha} \cos \left(\varphi_{1 \alpha}\right)+2 X_{\alpha 0} \sin \left(\varphi_{1 \alpha}\right)}
$$

with

$$
X_{\alpha 0}=\frac{R_{1 \alpha 0} \varphi_{1 \alpha 0} \cos \left(\varphi_{1 \alpha 0}\right)}{a_{\alpha 0}-c_{\alpha 0}}
$$

Where $S_{i \alpha}, R_{i \alpha}, \varphi_{2 \alpha}, Y_{\alpha}$ are current geometrical parameters (Figure 6) that can be expressed according to $\varphi_{1 \alpha}, X_{\alpha 0}$ and $a_{\alpha}$.

The previous equations can be brought to the resolution of a two nonlinear equation system, using a Newton method. Results obtained with this model are consistent with the experimental results. They are very good at low strains. These results are presented in Figure 7. A simpler model adapted from Kawabatta's has also been developed [5]. Results are once again consistent with experimental results $[6,16]$ but this simplified model nevertheless shows its limits. More specifically, the interpolation of the middle thread by the articulated straight segments does not prevent

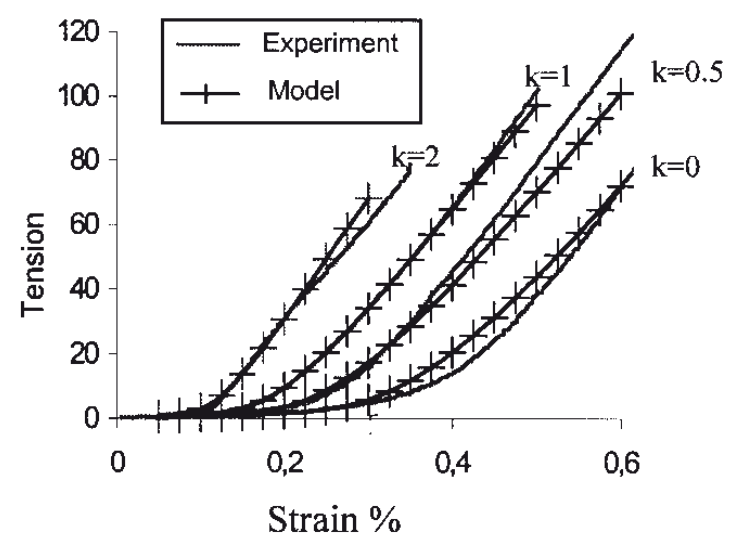

Figure 7. Comparison model/experiment for a balanced plane weave. 
geometric penetration of the thread networks. Moreover, the identification of the transverse behavior rule leads to parameters whose values are not consistent with physics.

\section{NUMERICAL SIMULATIONS AT MESO SCALE}

Local information is difficult to be obtained experimentally. The 3D simulations at mesh scale have two aims. First, it goals at analyzing the micro and meso nonlinear phenomena influencing global behavior. Moreover, it can allow defining the characteristics of a fabric prior to design, from its components and weaving.

Because of the nature of the yarn, made of many fibers, and the resulting lack of most stiffness, the problem is not classic. Calculations are carried out in the field of great deformations. The chosen law is hypoelastic type. In order to take into account the absence of some stiffness (in bending, shear and compression), it was shown that shear moduli and Poisson's ratios must be equal to zero, and that transverse tensile moduli are negligible front of tensile modulus in the yarn direction. But nil or nearly nil values of some mechanical coefficients leads to numerical difficulties when used in simulations. This is a phenomenon similar to the hourglass modes problems encountered in the building of reduced-integration elements. Those modes are, here, controlled by adding artificial stiffness that doesn't influence the solution. To take into account the possible displacement between the yarns, the contact with friction is introduced with a master-slave approach. Otherwise, Young's moduli in transverse direction being very different of the one in yarn direction, directions of anisotropy have to remain strictly in material directions (the ones of yarns). This condition is fulfilled thanks to reinforcements fixed to the elements in the yarn direction. The longitudinal modulus is identified from a tensile test on a yarn. The transverse modulus is given by the crushing law of the following form [17]:

$$
E_{3}=E_{\varepsilon}+E_{0}\left|\varepsilon_{33}^{n}\right| \varepsilon_{11}^{n}
$$

$E_{0}, m$ and $n$ are three material parameters identified with a biaxial test $k=1$, using an inverse method. Simulations carried out on a plain weave fabric pattern with symmetry boundary conditions made of glass fibers (Figure 8) show a good agreement with experiments (Figure 9). Two local phenomena can be observed:

- important undulation changes,

- a crucial role played by the transverse compressive behavior: the yarn flattening can reach, values up to $40 \%$ (Figure 8). 

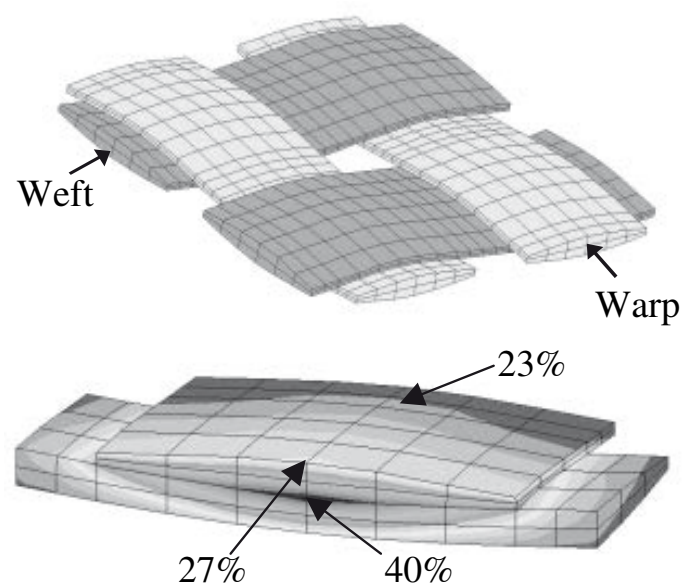

Figure 8. Plain weave mesh and transverse strain for a biaxial test $k=1$.

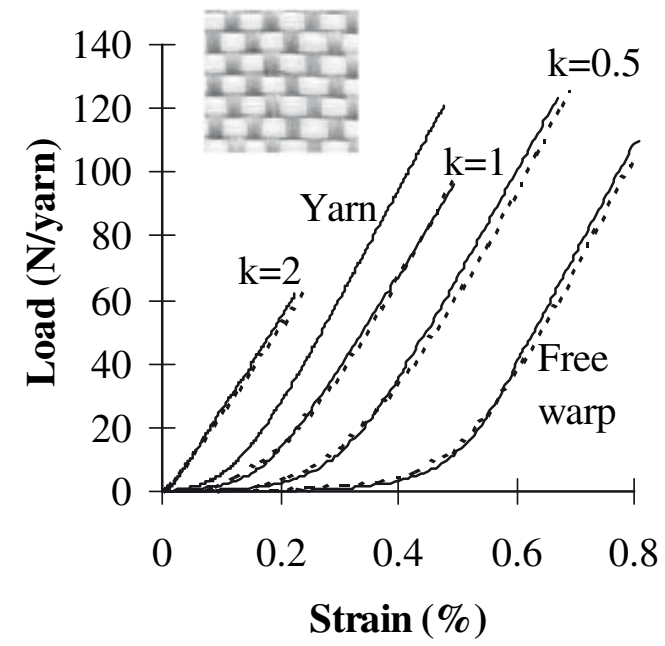

Figure 9. Comparison tests (solid lines)/simulations.

The study has been performed for other weaving patterns. It is also possible to see the influence of some geometrical and mechanical parameters [17].

\section{CONCLUSION}

The specific constitution of fabrics and yarns implies any other stiffness be negligibly compared to the tensile one. The mechanical behavior of fabrics is then represented by the relation between the weft and warp tensions and strains. A biaxial tensile device has been designed and allows to determine experimentally this biaxial tensile law for any fabric. Two numerical models of the biaxial behavior of fabrics can then be identified: a 3D finite element model of the pattern, that gave a lot of interesting local data, and a meso/macro consistent model, the aim of which is to simulate the behavior of the fabric in a finite element code. Both of them show results that are consistent with the experimental results. 


\section{ACKNOWLEDGMENT}

\section{This research is partially supported by the Aerospatiale-Matra Company.}

\section{REFERENCE}

1. Carronnier, D. and Gay, D. (1996). Approche intégrée du RTM. Revue des composites et des matériaux avancés, Hermés. 6.

2. Maison, S., Thibout, C., Garrigues, C., Garcin, J.L., Payen, H. and Sibois, H. (1998). Technical developments in thermoplastic composites fuselages. SAMPE journal, 34-35.

3. De Luca, P., Lefebure, P., Pickett, A.K., Vodermayer, A.M. and Werner, W. (1996). The numerical simulation of some press forming of continuous fiber reinforced thermoplastics. In: Proceedings of the 28th International SAMPE Technical conference. Seattle.

4. Van Der Ween, F. (1991). Algorithms for draping fabrics on doubly curved surfaces. International Journal of Numerical Method in Engineering, 31: 1414-1426.

5. Kawabata, S. (1989). Nonlinear mechanics of woven and knitted materials. Textile Structural Composites, Elsevier, 3: 67-116. In: Chou, T.-W. and Ko, F.K. (eds.).

6. Launay, J., Buet, K., Hivet, G. and Boisse, Ph. (1999). Analyse expérimentale et modéles pour le comportement mécanique biaxial des renforts tissés de composites. Revue des Composites et des Matériaux Avancés, 9(1): 27-56.

7. Boisse, P., Borr, M., Buet, K. and Cherouat, A. (1997). Finite element simulation of textile composite forming including the biaxial fabric behaviour. Composites: Part B, Elsevier, 28B: $453-464$.

8. Aubourg, N. and Mion, D. (1989). Etude de la Modélisation de l'emboutissage d'un tissu de fibres de verre. PSA/Ecole Polytechnique Report .

9. Ishikawa, T. and Chou, T. (1983). Non linear behaviour of woven fabric composites. Journal of Composite Materials, 17: 399-413.

10. Lene, F. (1990). Technique d'homogénéisation des composites à renforts tissés. Mécanique Matériaux Electricité, 433: 24-28.

11. Ladevéze, P., Gasser, A. and Allix, O. (1994). Damage mechanisms modeling for ceramic composites. Journal of Engineering Materials and Technology, 116: 331-336.

12. Realf, M.L., Boyce, M.C. and Backer, S. (1993). A micromechanical approach to modelling tensile behaviour of woven fabrics. Use of Plastic and Plastic Composites: Materials and Mechanics Issues, Stockes 46: 285-293.

13. Boisse, P., Buet, K., Gasser, A. and Launay, J. (2001). Meso-macro mechanical behaviour of textile reinforcements of thin composites. Composites Science and Technology, 61(3): 395-401.

14. Boisse, P. and Borr, M. (1996). Etude expérimentale du comportement mécanique biaxial des tissues. CRAS, Paris, 323: 503-509.

15. Borr, M. and Boisse, P. (1995). Détermination du comportement biaxial des tissus de fibres de verre. Mesure optique de la déformation. presented at Photomécanique 95. Cachan, 187-194.

16. Buet Gautier, K. (1998). Analyse et simulation du comportement mécanique des renforts composites tissés. Thése de Doctorat, Université d'Orléans.

17. Gasser, A., Boisse, P. and Hanklar, S. (2000). Analysis of the mechanical behaviour of dry fabric reinforcements. 3D simulations versus biaxial tests. Comp. Material Science, 17(1): $7-20$. 